\title{
Formación docente a nivel de postgrado en Latinoamérica
}

\section{Teacher training at the graduate level in Latin America}

\author{
DOI: http://dx.doi.org/10.17981/cultedusoc.10.2.2019.08
}

Recibido: Abril 23 de 2019 Aceptado: septiembre 13 de 2019

Jhan Carlos Herrera Pérez

Universidad de Baja California, Tepic (México)

stickmaster2005@gmail.com

Para citar este artículo:

Herrera, J. (2019). Formación docente a nivel de postgrado en Latinomáerica. Cultura, Educación y Sociedad, 10(2). 97-108. DOI: http:// dx.doi.org/10.17981/cultedusoc.10.2.2019.08

\section{Resumen}

El artículo de reflexión se orienta a analizar la formación docente a nivel de postgrado en el contexto latinoamericano, contextualizado principalmente en estudios de Maestría y Doctorado, Se enfatiza la importancia de la investigación como un componente esencial de estos programas académicos y los retos que se deben plantear actores como: gobierno, instituciones y docentes para contribuir al mejoramiento de la calidad educativa. Desde el fundamento metodológico un análisis reflexivo-argumentativo permite la construcción teórica en atención a las principales categorías trabajadas: Formación Docente - Estudios de Postgrado - Calidad Educativa. Entre los principales resultados destaca que los diferentes gobiernos intentan establecer mecanismos que les permitan mejorar el control del presupuesto, pero en sus afán por lograr objetivos referidos por organismos internacionales como la Organización para la Cooperación y el Desarrollo Económico (OCDE), establecen políticas que no son concertadas con todos los actores, incluidos gremios o sindicatos, lo que ha derivado en consecuencias negativas para los educadores. Se concluye que la formación docente a nivel de postgrado, contribuye con el mejoramiento de la calidad educativa por cuanto fortalece: el perfil de competencias, la innovación didáctica - pedagógica y la promoción en el escalafón docente.

Palabras clave: Formación docente; estudios de postgrado; calidad educativa

\section{Abstract}

It emphasizes the importance of research as an essential component of these academic programs and the challenges that must be posed by actors such as government, institutions, and teachers in order to contribute to the improvement of educational quality. From the methodological foundation, a reflective-argumentative analysis allows the theoretical construction in attention to the main categories worked: Teacher Training - Postgraduate Studies - Educational Quality. Among the main results, it stands out that the different governments try to establish mechanisms that allow them to improve the control of the budget, but in their eagerness to achieve objectives referred to by international organizations such as the Organization for Economic Cooperation and Development (OECD), they establish policies that are not agreed upon with all the actors, including unions, which has resulted in negative consequences for educators. It is concluded that teacher training at the postgraduate level contributes to the improvement of educational quality by strengthening: the profile of competencies, didactic-pedagogical innovation, and promotion at the teacher level.

Keywords: Teacher training; postgraduate studies; educational quality 


\section{INTRODUCCIÓN}

Uno de los aspectos fundamentales para mejorar la calidad educativa es la formación de los docentes; en las últimas décadas, ha crecido la demanda de estudios de postgrados por parte de los docentes de los diferentes países latinoamericanos, esto en gran medida a los estímulos que se generan desde los nuevos estatutos sobre carrera docente, así como la necesidad de mejorar las estrategias pedagógicas y disciplinares, de esta forma lograr en los estudiantes mejores resultados en las evaluaciones institucionales y nacionales; sin lugar a duda aún quedan temas pendientes que se convierten en grandes retos para los actores que intervienen en este sector tan importante de la sociedad como lo es la educación.

$\mathrm{Al}$ respecto, es una realidad que el nivel de desarrollo de un país se nota sustancialmente en la calidad de educación; no es una simple coincidencia que los países a la vanguardia de los s avances científicos y tecnológicos y transformaciones significativas en sus sistemas educativos evidencie importantes grados de desarrollo económico y social. Se requiere mayor inversión por parte gubernamental para alcanzar una mayor cobertura en todos los niveles y modalidades, además de asegurar el aprendizaje y la calidad.

Consecuentemente, este artículo se orienta a analizar la formación docente a nivel de postgrado en el contexto latinoamericano, contextualizado principalmente en estudios de Maestría y Doctorado. Por tanto, destaca la investigación como un componente esencial de estos programas académicos, a través de la participación del gobierno, las instituciones y docente en pro de la calidad educativa.
Rol del docente en el aseguramiento de la calidad educativa

En el último decenio del siglo pasado el sistema educativo de diferentes regiones del mundo, incluidos los de América Latina han definido políticas que evidencian esfuerzos tendentes al mejoramiento de los indicadores de calidad educativa. Una de las principales dimensiones para el análisis está representado por el desempeño profesional del maestro, como una categoría influyente para lograr un salto cualitativo de la gestión escolar. Así mismo, entre las múltiples acciones que pueden realizarse para el mejoramiento de la calidad de la educación, la evaluación del docente juega un papel de primer orden, pues permite caracterizar su desempeño y por tanto orientar las acciones para propiciar su desarrollo futuro; constituye una vía fundamental para su atención y estimulación (Schulmeyer, 2002).

Desde la perspectiva del rol de los docentes en atención a la importancia de resignificar su trabajo y recuperar su centralidad supone abrirse al reconocimiento que hay un conjunto de factores que determinan el desempeño. Entre ellos: formación inicial, desarrollo profesional en servicio, condiciones de trabajo, salud, autoestima, compromiso profesional, clima institucional, valoración social, capital cultural, salarios, estímulos, incentivos, carrera profesional, evaluación del desempeño (Robalino, 2005). Procesos como la actualización curricular, mejoramiento de la equidad y la calidad, descentralización en las modalidades de gestión, deberán hacerse acompañar de estrategias conducentes al fortalecimiento del perfil docente como agente de cambio en el ámbito social. 
Es necesario, en consecuencia, lograr reformas significativas, efectivas y ante todo sustentables en las prácticas profesionales y culturas de trabajo de los docentes. Así como el sistema requiere la actualización en planes de estudio, disposición de recursos educativos, mejoramiento de la infraestructura física, también debe focalizarse en el fortalecimiento de capacidades en los docentes que permitan desplegar un perfil de competencias que contribuyan al perfeccionamiento educativo (Vaillant, 2005).

En su práctica cotidiana, el maestro se enfrenta a sus estudiantes y sus expectativas, tan disímiles como el número de aprendices en el aula, y se compromete con las particularidades de cada uno. No sólo el saber de cada estudiante es diverso sino sus modos y fuentes de motivación hacia el aprendizaje. Es importante, entonces, que conozca estas diferencias para procurar el aprendizaje de todos y cada uno. A partir de esas particularidades, los maestros diseñan sus clases y adaptan los materiales de acuerdo con las necesidades y los retos pedagógicos a los que se enfrentan. Todo ello para formar hombres y mujeres con las capacidades y conocimientos necesarios para participar activamente en su sociedad (MIN, 2005).

Se enseña para hacer posible la apropiación y el disfrute de la riqueza cultural, a fin de que los que aprenden puedan satisfacer sus necesidades materiales y simbólicas, para desarrollar su sensibilidad en el goce de las creaciones humanas y construir una sociedad más justa (Hernández, 2005).

La profesión docente está cargada de matices, unos despiertan la pasión por la enseñanza, pero otros pueden desmotivar a sus actores. Algunos están relacionados con factores externos y otros con factores internos y más personales. En unos influyen los ideales y en otras la realidad. Sin menoscabar aquellos valores más intrínsecos como la vocación, en la enseñanza existen múltiples factores que motivan a este colectivo; destacan entre otros: el salario, las vacaciones, el trato con los niños, los horarios laborales (Sánchez, 2009). De tal manera que el ejercicio docente está influenciado de forma significativa por variables cuya dinámica prescribe una dinámica de relaciones en el ámbito intra e intersistema.

Importancia de los estudios de postgrado en la formación docente

Hoy en día, es vital que los docentes se capaciten, que sus conocimientos satisfagan las necesidades de las nuevas generaciones. Por lo anterior, la psicología como la ciencia que estudia el comportamiento ve los procesos formativos el medio a través del cual el docente desarrolla un aprendizaje y nuevas habilidades que posteriormente con la práctica se convertirán en competencias a ser transferidas en sus ámbitos de actuación con sus estudiantes (Moreno, 2009). En este sentido, Mena y Huneeus (2017), coinciden en afirmar que el aprendizaje requiere un contexto social en donde se fortalecen procesos convivenciales, esto significa que la praxis docente representa un espacio idóneo para el desarrollo de competencias y habilidades.

Para que un docente tenga posibilidades de asegurar modificaciones en el desempeño académico de sus estudiantes, debe tomar en cuenta que los logros en el aprendizaje están asociados al desarrollo del trayecto escolar íntegro del alumno. Ruta definida en correspondencia con la organización y el ambiente escolar, así como 
con los propósitos y acciones del colectivo docente completo de la escuela (Peña y Ochoa, 2012). Se traducen procesos educativos orientados a la formación integral del ciudadano; al respecto, Marín, Cabas, Cabas y Paredes (2018), señala que indistintamente del nivel educativo, la preparación académica estará fundamentada en principios de integralidad, pertinencia y contextualización.

En Latinoamérica, más específicamente en Colombia, el Ministerio de Educación Nacional (MEN, 2014), establece diferencias sustantivas en cuanto al propósito de la formación de postgrado; así plantea que los estudios de maestría en educación orientan la profundización de las relaciones entre: aprendizajes-enseñanzas, pedagogías-didácticas; sujetos de la educación, problemas de la educación y contextos culturales-diversidad, que desde la investigación multimodal redefinan el lugar de la producción de saberes pedagógicos. Ello dará lugar a la comprensión de la diversidad de formas para ser integradas en el mundo de los sujetos de la educación, que exigen el reconocimiento de las diferencias y la unidad en la diversidad. Este propósito conducirá necesariamente hacia un desempeño profesional de alta calificación con niveles de profundización teórica superiores a los de la especialización en todas las dimensiones.

Por su parte los doctorados y posdoctorados, dentro del Sistema colombiano de formación de educadores, comportan un reto aún más alto, el de la construcción de teoría pedagógica que movilice la innovación creativa del conocimiento y circulación de saber pedagógico orientado a la transformación educativa, a la generación de modelos propios para los múltiples contextos colombianos, a partir de estudios investigativos de alto valor pedagógico, que realimenten de manera permanente los horizontes de la formación docente. En aras de una educación de calidad, para un país multiétnico y multicultural como Colombia, deben exigirse comprensiones permanentes de los procesos educativos que atiendan las condiciones sociopolíticas (MEN, 2014).

\section{La formación de posgrado en el fortalecimiento de la carrera docente}

Según la UNESCO (2013) los programas de formación docente existentes en América Latina y el Caribe han concentrado su atención en la carrera docente, es decir que el hecho de ascender en el escalafón propuesto en los diferentes sistemas educativos es visto como una de las metas más importantes a alcanzar a través de estos programas. También resalta la proyección social o el desarrollo profesional del maestro, en relación con su rol transformador de la sociedad en general, tiene implicaciones en las acciones y estrategias que emprende para mejorar el sistema escolar de acuerdo a las necesidades de cada contexto. Los fundamentos teórico - normativos, deben centrar igualmente la discusión, en el desarrollo de habilidades, manejo de información, conocimiento, desarrollo profesional, investigación, innovación y desarrollo, como componentes esenciales en estos procesos formativos (Herrera, Vega, Barrios, Darcy, Díaz y Garzón, 2015). En Colombia solo el $28 \%$ de los docentes vinculados en el sector oficial tiene estudios de posgrado (especialización y maestría), frente al $71 \%$ conformado por licenciados o profesionales con diferente formación, que certifican estudio de pregrado (MEN, 2015).

La preocupación por mantener a los profesores motivados mediante un reconocimiento externo es general en todos los 
países de América. Dos son las estrategias diseñadas para lograrlo. Por una parte, lo que se puede denominar como promoción horizontal, por la cual los docentes ven reconocido su trabajo mediante incrementos salariales o ascensos en un escalafón, sin que ello suponga modificar sus labores de docente de aula o asumir mayores responsabilidades. Y, por otra parte, mediante la promoción vertical por la cual los docentes ascienden laboralmente asumiendo tareas directivas o de supervisión, siempre con incremento salarial. Los países de América Latina cuenta con un sistema de escalafón docente con una serie de categorías profesionales para reconocer el trabajo de sus profesores. Este escalafón puede servir para determinar tanto los complementos salariales como otros beneficios laborales (prioridad en el cambio de escuela, por ejemplo) o profesionales (prioridad para licencias por estudios o elección de horarios) (Murillo, Gonzales y Rizo, 2006).

Para Robalino (2005), los sistemas de promoción o escalafonamiento contribuyen a elevar la calidad docente, así como también, mejoran la motivación y el interés. Igualmente, en el caso de los sistemas de promoción vertical, buscan seleccionar a los mejores candidatos para el ejercicio de tareas directivas o de supervisión" (Robalino, 2006). La formación posgradual se convierte en una de las condiciones para mejorar en la carrera profesional, ascenso para el escalafón, así como también, mejorar al reconocimiento social del docente. En el caso específico de Colombia la promoción es de tipo horizontal, es decir se asciende dentro del mismo cargo ya sea docente o directivo docente.

Así, el Estatuto Docente (Decreto Ley 1278, 2002), establece como uno de los requisitos para alcanzar el grado 3 "poseer título de maestría o doctorado en un área afín a la de su especialidad o desempeño o en un área de formación que sea considerada fundamental dentro del proceso de enseñanza aprendizaje de los estudiantes", lo cual hace que, además del interés por el desarrollo profesional, también exista un interés relacionado con mejores ingresos para los docentes que cuentan con estudios de maestría. No obstante, el acceso a esta formación resulta difícil para los docentes por los costos que representa esta inversión (MEN, 2015).

\section{Orientación de la investigación en la formación de postgrado}

Los programas académicos de postgrado incluidos los orientados a la formación docente integran a sus currículos el componente de investigación, como una característica asociada a sus concepciones y dinámicas. Se incluyen cursos y módulos referidos a métodos de investigación y sus diversas variantes (experimentación, encuestas, análisis históricos). Los cursos incluyen seminarios sobre el uso de la evidencia a lo largo del ejercicio de la profesión. Los maestros y profesores reciben una fuerte preparación en la investigación del comportamiento de niños y adolescentes. Además, la investigación está muy presente en la práctica guiada que los futuros docentes realizan en las escuelas, donde tienen la oportunidad de analizar el desempeño y la conducta de los estudiantes, el clima en el centro educativo y el relacionamiento con las familias. Deben reflexionar, extraer conclusiones y proponer líneas concretas de acción (Vaillant y Manso, 2012).

Por su parte, Pineda, Uribe y Díaz (2007), conceptualizan la enseñanza de la investigación en la formación de posgrado de docentes como una actividad 
compleja, donde el contexto juega un rol determinante; se pueden generar situaciones problemas, que, en su mayoría, son imprevistos y que demandan opciones éticas y políticas del profesor. Formar al estudiante para la investigación dentro de esta perspectiva, significa considerar la práctica como principio rector del aprendizaje, y al profesor como el recurso más eficaz para que el estudiante desarrolle sus capacidades y sus propias experiencias.

De igual forma, las estrategias de enseñanza, entendidas como mediación que posibilita la formación para la investigación, deben caracterizarse por permitir al estudiante observar de manera sistemática las situaciones del contexto del que forman parte, analizarlas con fundamentos teóricos; discutir conceptual y metodológicamente los condicionantes y las formas de generar conocimiento a partir del diagnóstico de los problemas y del diseño de estrategias que posibiliten su transformación.

\section{La necesidad de fortalecer los procesos formativos en estudios de postgrado}

La evolución reciente de la demanda docente hacia los postgrados plantea nuevos desafíos. Entre ellos se destaca la necesidad de incorporar criterios de pertinencia y potencial de impacto en las prácticas de enseñanza; y la entrega de becas para estudios en áreas prioritarias, considerando los méritos de los docentes y las necesidades de sus centros educativos (UNESCO, 2013), esto traduce el reto de fortalecer el sentido de pertinencia en la oferta formativa de los programas académicos de posgrado.

En Latinoamérica, más específicamente de acuerdo con la información disponible sobre México, el cuerpo docente se inclina en una proporción significativa a continuar estudios de postítulo, por su parte en Perú, hay una tendencia similar a ofrecer, demandar y cursar estudios de postgrado, no existiendo una relación directa entre la disciplina que enseña el docente y la especialización por la que opta en el postítulo (UNESCO, 2013). Se infiere que la formación docente es estudios de postgrado debería redundar en un mejoramiento de la calidad educativa y el aseguramiento del aprendizaje. Sin embargo, el problema de la calidad, la cobertura y la equidad en la educación se ha presentado de manera consistente en el sistema educativo; destaca que, la formación de posgrado de los docentes no es un factor condicionante de las buenas prácticas escolares en la educación obligatoria (Sandoval, 2015).

El análisis de la oferta educativa de educación superior en países como México, permite percibir que el crecimiento más dinámico en la última década es el del posgrado, porque éste se dio con intensidad y características similares a las que en su momento tuvo la expansión del nivel licenciatura en la década de los años 70: en respuesta a la presión de los demandantes, sin contar con plantas docentes suficientes y sólidamente preparadas, en algunos casos improvisando programas e instituciones para ofertarlos (Moreno, 2003).

Tanto en Iberoamérica como en Latinoamérica los procesos de capacitación que reciben los profesores por parte del estado no responden a las necesidades de la docencia en el nivel básico (Martín, Manso, Pérez y Álvarez, 2010). Con frecuencia los docentes manifiestan que son obligados a asistir a cursos, sin que se realice un diagnóstico de necesidades de capacitación. Un extenso número de estudios sobre 
la Educación Superior en América Latina y el Caribe (Didriksson y Gazzola, 2008) se ha referido a la expansión desordenada de la oferta de estudios de postgrado (diplomados, especializaciones, maestrías y doctorados) y ha demostrado que esta eclosión, particularmente visible en aquellos sistemas de educación superior con una alta participación de instituciones privadas sujetas a registros de validez laxos, tiene una naturaleza reactiva y se ha desarrollado sin apego a reglas de calidad y pertinencia (UNESCO, 2013).

Los sistemas nacionales de acreditación y certificación, en principio, respaldan la calidad de los programas de postgrado ofrecidos por instituciones de educación superior, de acuerdo con los parámetros internacionalmente aceptados. Hay que reconocer que la experiencia predominante atañe a la preparación para la investigación fundamental, lo que es positivo en términos de la solidez institucional del oferente. Pero, si se pretende impulsar modelos de postgrado relevantes, que enfaticen los componentes de la formación práctica, centrales para la modificación de las prácticas de enseñanza, es insoslayable desarrollar criterios e indicadores complementarios que permitan una criba más fina, centrada en la pertinencia y el potencial de impacto (Darling-Hammond, 2006). Regular la oferta de programas de postgrado en educación, con base en referentes de calidad y pertinencia, que aún no están suficientemente desarrollados ni son compartidos por la comunidad, es una tarea urgente en la región (UNESCO, 2013).

En líneas generales se asume que, los docentes en su mayoría desarrollan programas de formación de postgrado con base a intereses personales. Además, aunque se reconoce el esfuerzo de las políticas de estado por mantener esta estrategia y crear condiciones para mejorar la formación de los maestros se hace necesario facilitar espacios concretos para llevar a cabo procesos de investigación educativa, en donde colectivos de maestros y maestras puedan enriquecer su saber pedagógico y aportar en las reformas que la educación necesita (Herrera et al, 2015).

La enseñanza puede verse fortalecida por la formación en investigación, por ello los esfuerzos encaminados a ofrecer maestrías en educación y administración educativa, deben conectarse a la necesidad de apoyo financiero y tiempos para su desarrollo, con el fin de evitar o reducir el número de docentes que una vez logrado el nivel de magíster o doctor abandonen la escuela para ubicarse en universidades (Herrera et al, 2015). También destaca que los diferentes gobiernos intentan establecer mecanismos que les permitan mejorar el control del presupuesto, pero en sus afán por lograr objetivos referidos por organismos internacionales como la Organización para la Cooperación y el Desarrollo Económico (OCDE), establecen políticas que no son concertadas con todos los actores, incluidos gremios o sindicatos, lo que ha derivado en consecuencias negativas para los educadores. Hay factores de tiempo y coordinación en los colegios que dificultan que los docentes puedan asistir a los cursos de formación (García, Maldonado, Perry, Rodríguez y Saavedra, 2014).

Algunas de las razones por las cuales se ha incrementado el número de postgrados en la región se exponen a continuación:

a. La crisis económica ha traído consigo la disminución en las ofertas laborales, lo que a su vez incide en el aumento de cualificación profesional como requisito, tanto para la obtención de empleo, como para la permanencia o el ascenso en el mismo (Moreno, 2003). 
b. Los sistemas de promoción laboral y salarial establecidos para los académicos universitarios y los profesores de educación básica y normal, orientan fuertemente a los profesionales de la educación, e incluso a los de otras disciplinas, a buscar vías para el fortalecimiento del curriculum profesional (entendido más como certificación que como desempeño o productividad), entre ellas, la obtención de grados posteriores a la licenciatura. En muchos casos, tanto las instituciones que demandan a sus profesores la obtención de grados académicos, como los mismos aspirantes, encuentran que la vía más rápida, fácil y segura es la de los posgrados en educación (Moreno, 2003).

c. El exceso de aspirantes a los programas de posgrado en educación genera a su vez una fuerte presión a las instituciones que los ofertan, muchas de las cuales optan por responder a la demanda sin contar con las condiciones académicas y de infraestructura que requiere este nivel educativo (Moreno, 2003).

En las circunstancias antes descritas, las prácticas de formación (orientadas a la investigación o a la profesionalización) en los programas de posgrado resultan, en algunos casos, improvisadas; buen número de los académicos responsables de la formación no tienen una trayectoria profesional y/o de investigación que avale y sustente su tarea formadora $\mathrm{y}$, por otra parte, muchos de los demandantes ingresan a los posgrados en educación más por la oportunidad que como expresión de un genuino interés por la investigación educativa, por la superación profesional y/o por una carrera académica (Moreno, 2003).

Es impostergable un repensamiento profundo de los posgrados en educación; dicho en otras palabras, es necesaria una recreación innovadora de los mismos, en cuya posibilidad de logro habrá de jugar un papel importante la voluntad política de todos los niveles y ámbitos de autoridad, la toma de conciencia y el decidido apoyo de los líderes gremiales, así como la sensibilización de los equipos que asumen la toma de decisiones en los ámbitos de planeación y presupuesto educativo en cada cambio de administración para evitar que, tanto la investigación educativa, como los posgrados en educación, se ubiquen en forma desventajosa en el panorama de las urgencias educativas (Guadalupe y Bayardo, 2002).

La acción innovadora en los posgrados en educación tendría entre sus metas la búsqueda de la consolidación en todos los aspectos: académico, de valoración en el posgrado nacional, de sensibilización presupuestal, de métodos de trabajo pertinentes, de formas de organización en las que el tiempo de atención a los estudiantes no se reduzca al mínimo, de integración y conformación de cuerpos académicos dedicados al posgrado, así como de fortalecimiento de la infraestructura de apoyo académico (Guadalupe y Bayardo, 2002).

Se percibe una tendencia internacional de intervención de los gobiernos a las políticas de los posgrados. A través del condicionamiento de los recursos, se presiona por organizaciones nacionales e internacionales, para que los posgrados lleven a cabo dispositivos de evaluación, acreditación y mejora en la eficacia y racionalidad en los procesos y productos (Ponce, 2003).

Estos criterios son, sobre todo, de eficacia en la eficiencia terminal, de pertinencia social y económica, adecuada a los procesos tecnológicos y venta de los servicios.. Otras dificultades consisten en la falta de flexibilidad curricular y lentitud para adaptarse 
a los cambios. Muchos programas carecen de estrategias para evaluar la pertinencia social de sus ofertas académicas (Ponce, 2003). Se presiona sobre todo para reducir los tiempos de transferencia del conocimiento producido en la investigación a la solución de problemas concretos del ámbito socioeducativo.

\section{Conclusiones}

En los últimos años la mayor parte de los países de la región ha fortalecido sus programas de apoyo a la formación de posgrado, se realizan esfuerzos significativos por evidenciar un crecimiento sostenido en sus tasas de egreso. Los propósitos expresos de estas políticas parten de la premisa fáctica de una insuficiencia en la dotación de personal altamente calificado, que funciona como un obstáculo significativo para el despliegue de los sistemas de innovación (Albornoz, 2010).

Se hace necesario profundizar en el análisis acerca de quienes están formando a los docentes de educación media a nivel de postgrado, lo que repercute en la educación recibida por los estudiantes en los diferentes colegios de Latinoamérica. Asimismo, es necesario fomentar una cultura sostenible de acreditación de los postgrados que atienda a los intereses y necesidades crecientes de los países y de la región, capaz de generar confianza entre los sistemas nacionales de educación superior (Pires y Lemaitre, 2008).

Los problemas del posgrado además son persistentes. Para (Álvarez, 2002) existen núcleos asociados a desequilibrios matriculares en relación a las disciplinas o áreas del conocimiento, la necesidad de fortalecer los procesos de investigación, así como también el talento humano responsable de la mediación en estos programas formativos, la relación ingreso - prosecución - egreso, la pertinencia de los programas formativos en relación a los requerimientos contextuales y la vinculación con sectores sociales y productivos.

Sin lugar a dudas la carrera docente ha motivado a los docentes a cursar un postgrado con el objeto de ascender, los políticas gubernamentales deben viabilizar el acceso de la mayoría de la población docente, sobretodo la rural, para ello deben crear incentivos económicos, como becas y créditos, además de fortalecer los programas de acreditación que debe traer consigo una vinculación intrínseca entre lo que se enseña en las aulas universitarias y lo que se vive en la cotidianidad en las instituciones educativas.

\section{REFERENCIAS}

Albornoz, M. (2010). Formación de posgrado en américa latina. Políticas de apoyo, resultados e impactos. Buenos Aires: Eudeba. Disponible en https:// observatoriocts.oei.org.ar/files/Archivo\%20Documental/Libros\%20del $\% 20$ Observatorio/formacion_de_postgrado.PDF

Álvarez, G. (2002). La calidad y la innovación en los posgrados. Revista de la Educación Superior. Asociación Nacional de Universidades e Instituciones de Educación Superior (ANUIES), (124), 31-38. Disponible en http://publicaciones.anuies.mx/revista/124/3/2/es/la-calidad-y-la-innovacion-en-los-posgrados

Darling-Hammond, L. (2006). Assessing Teacher Education: the usefulness of multiple measures for assessing program outcomes. Journal of Teacher Education. 57(2), 120-138. https://doi. org/10.1177/0022487105283796 
Didriksson, A. y Gazzola, A. (Ed.) (2008). Tendencias de la educación superior en América y el Caribe. En, UNESCO, Conferencia Educación Superior para Todos, Instituto Internacional para la Educación Superior en América Latina y el Caribe (IESALC), Caracas, Venezuela. Disponible en https:// unesdoc.unesco.org/ark:/48223/ pf0000161990

García, S., Maldonado, D., Perry, G., Rodríguez, C. y Saavedra, J. (2014). Tras la excelencia docente. Cómo mejorar la calidad de la educación para todos los colombianos. Bogotá D.C.: Puntoaparte. Disponible en www.fundacioncompartir.org/pdf/ Traslaexcelenciadocente18.02.2014. pdf.

Guadalupe, M. y Bayardo, M. (2002). Innovación en los posgrados en educación. ¿Sólo un caso particular de lo posible? Higher Education Journal Issue Number, (124), 73-84. Disponible en http://publicaciones.anuies.mx/ pdfs/revista/Revista124_S3A5ES. pdf

Hernández. C. (2005). Para ejercer el oficio de maestro. Altablero, 34. [Online]. Disponible en https://www.mineducacion.gov.co/1621/article-87110. html

Herrera, J., Vega, V., Barrios, Darcy M., Díaz, A. y Garzón, J. (2015). El lugar de la investigación en la formación posgradual de los docentes del distrito en la Bogotá humana. Bogotá, D.C.: Institución para la Investigación y el desarrollo pedagógico. Disponible en https://repositorio.idep. edu.co/handle/001/832
Marín, F., Cabas, L., Cabas, L. C. y Paredes, A. J. (2018). Formación Integral en Profesionales de la Ingeniería. Análisis en el Plano de la Calidad Educativa. Formación Universitaria, 11(1), 13-24. http://dx.doi.org/10.4067/S071850062018000100013

Martín, E., Manso, J., Pérez, E. y Álvarez, N. (2010). La formación y el desarrollo profesional de los docentes. Madrid: Fuhem. Recuperado de http://www.fuhem.com/media/ educacion/File/Encuesta\%202010/ Encuesta\%20Formacion\%20y\%20 Desarrollo\%20Profesional\%20Docente_FUHEM_2010.pdf.

Mena, M. y Huneeus, M. (2017). Convivencia Escolar para el aprendizaje y buen trato de todos: Hacia una mejor comprensión del concepto. Cultura Educación y Sociedad, 8(2), 9-20. https://doi. org/10.17981/cultedusoc.8.2.2017.01

Moreno, E. (2009). Evaluación de competencias docentes en espacios educativos del tercer semestre del plan de estudios de psicología 2004 (PEP04) de la Universidad de Sonora. [Tesis de Licenciatura]. Universidad de Sonora, Sonora, México.

Moreno, M. (2003). El posgrado para profesores de educación básica. Cuadernos de Discusión, (5). México. D.F.: SEP, SEByN Disponible en http://studylib. es/doc/2338147/c-5-d-el-posgrado-para-profesores

Murillo, F., González V. y Rizo, H. (2006). Evaluación del desempeño y carrera profesional docente. Un estudio comparado entre 50 países de América y Europa. Santiago de Chile: UNESCO. Disponible en https:// unesdoc.unesco.org/ark:/48223/ pf0000152934_spa 
Peña, M. y Ochoa A. (2012). La capacitación continua de los docentes de educación básica en Sonora, México, y la política pública de evaluación: el caso del curso básico nacional al inicio del ciclo escolar. Estudios Sociales. Revista de Alimentación Contemporánea y Desarrollo Regional, (2), 401-416. Disponible en: https://www.redalyc. org/articulo.oa?id=417/41724972018

Pineda, L., Uribe, L. y Díaz, E. (2007). ¿Cómo se entiende formar para la investigación en contextos de educación media y de educación superior? Revista Científica Guillermo de Ockham, 5(2), 11-22.

Pires, S., Lemaitre, M. (2008). Sistemas de acreditación y evaluación de la educación superior en américa latina y el caribe. [Online]. Disponible en https://www.researchgate.net/ publication/242558278_Sistemas_ de_acreditacion_y_evaluacion_de_ la_educacion_superior_en_America_ Latina_y_el_Caribe

Ponce, V. (2003). Tendencias, perspectivas y retos del posgrado y la investigación en Latinoamérica. $A n$ tología. Los retos del postgrado en la educación. Guadalajara, Jalisco. Disponible en https://es.scribd.com/ document/204715938/Tendencias Perspectivas-y-Retos-Del-Postgradoy-La-Investigacion-en-Latinoamerica República de Colombia. MEN. (2015). Colombia, la mejor educada en el 2025. Líneas estratégicas de la política educativa del Ministerio de Educación Nacional, [Online]. Disponible en https://www.mineducacion.gov. co/1759/articles-356137_foto_portada.pdf
República de Colombia. MEN. (2014). Sistema colombiano de formación de educadores y lineamientos de política. [Online]. Disponible en ht t p s:// www.mineducacion.gov.co/1759/articles-345485_anexo1.pdf.

República de Colombia. MEN. (2005). Ser maestro hoy. El sentido de educar y el oficio docente. Altablero, 34. [Online]. Recuperado de https://www.mineducacion.gov.co/1621/propertyvalue-31232.html

República de Colombia. MEN. (junio 19 de 2002). Estatuto de Profesionalización Docente. [Decreto Ley 1278]. DO: 44.840. Recuperado de https:// www.mineducacion.gov.co/1621/articles-86102_archivo_pdf.pdf

Robalino, M. (2006). Carrera y Evaluación Docente en América Latina. Una mirada desde una perspectiva renovada de la profesión docente. Docencia, (29), 69-78. Disponible en http:// www.opech.cl/_doc/carrera_y_evaluacion_docente_en_america_latina_robalino.pdf

Robalino, M. (2005). ¿Actor o Protagonista? Dilemas y responsabilidades sociales de la profesión docente. Revista Prelac, 1(1), 6-23. Disponible en https://unesdoc.unesco.org/ ark:/48223/pf0000144666

Sandoval, F. (2015). La formación permanente del docente en Latinoamérica. Ride, 6(11), 1-17. Disponible en https://www.ride.org.mx/index.php/ RIDE/article/view/122

Sánchez, E. (2009). Dos caras de la carrera docente: satisfacción y desmotivación. Pedagogía Social. (16), 135-148. http://dx.doi.org/10.7179/ PSRI_2009.16.10 
Schulmeyer, A. (2002). Estado actual de la evaluación docente en trece países de América Latina. En, UNESCO, $E l$ Desempeño de los Maestros en América Latina y el Caribe: nuevas prioridades, Brasilia, Brasil. Disponible en http:// repositorio.minedu.gob.pe/bitstream/ handle/123456789/3092/30.\%20Estado $\% 20$ actual $\% 20$ de $\% 201$ a $\% 20$ evaluaci\%C $3 \%$ B $3 n \% 20$ docente $\% 20$ EN\%2013\%20PA\%C3\%8DSES\%20 DE\%20AM\%C3\%89RICA\%20LATINA.pdf? sequence $=1 \&$ isAllowed $=y$

UNESCO. (2013). Antecedentes y Criterios para la Elaboración de Políticas Docentes en América Latina y el Caribe. Santiago de Chile: UNESCO. Disponible en https://unesdoc.unesco.org/ ark:/48223/pf0000223249

Vaillant, D. (2005). Reformas Educativas y Rol de docentes. Revista Prelac, 1(1), 38-51. Disponible en https://unesdoc. unesco.org/ark:/48223/pf0000144711

Vaillant, D. y Manso, J. (2012). Tendencias en la formación inicial docente. Cuaderno de Investigación Educativa, 3(18), 11-30. https://doi.org/10.18861/ cied.2012.3.18.2703 